\title{
INVARIANT MEANS ON BANACH SPACES
}

\author{
RADOSEAW ŁUKASIK
}

\begin{abstract}
In this paper we study some generalization of invariant means on Banach spaces. We give some sufficient condition for the existence of the invariant mean and some examples where we have not it.
\end{abstract}

\section{Introduction}

Invariant means on amenable groups are an important tool in many parts of mathematics, especially in harmonic analysis (see [7, 8]). For basic properties of invariant means, we refer the reader to [7].

Invariant means and their generalizations for vector-valued functions play also an important role in the stability of functional equations and selections of set-valued functions (see [10, 3, 4, 11, 1]).

The space of all bounded functions from a group $(G,+)$ into a Banach space $X$ is denoted by $\mathcal{B}(G, X)$. Let us recall the definition of the amenable group.

Received: 29.01.2016. Accepted: 13.07.2016. Published online: 20.10.2016.

(2010) Mathematics Subject Classification: 43A07.

Key words and phrases: invariant mean, amenable semigroup, Banach space. 
Definition 1.1. A semigroup $(S,+)$ is called left [respectively right] amenable if and only if there exists a linear map $L: \mathcal{B}(S, \mathbb{R}) \rightarrow \mathbb{R}$ such that

$$
\begin{gathered}
\inf f(S) \leq L(f) \leq \sup f(S), \quad f \in \mathcal{B}(S, \mathbb{R}), \\
L\left({ }_{a} f\right)=L(f), \quad a \in S, f \in \mathcal{B}(S, \mathbb{R}) \\
{\left[L\left(f_{a}\right)=L(f), \quad a \in S, \quad f \in \mathcal{B}(S, \mathbb{R})\right],}
\end{gathered}
$$

where

$$
\begin{gathered}
{ }_{a} f(x)=f(a+x), \quad a, x \in S, f \in \mathcal{B}(S, \mathbb{R}) \\
{\left[f_{a}(x)=f(x+a), \quad a, x \in S, f \in \mathcal{B}(S, \mathbb{R})\right] .}
\end{gathered}
$$

If both left and right invariant means exist, then $S$ is called amenable.

REMARK 1.2. Every commutative semigroup is amenable.

Some generalization of invariant mean for vector-valued functions was investigated in [3] and [12] where we can find the following definition.

Definition 1.3. We say that a linear function $m: \mathcal{B}(G, X) \rightarrow X$ is an invariant mean if the following conditions hold:

(i) for every $f \in \mathcal{B}(G, X)$ and $a \in G$ there is

$$
m(f(a+\cdot))=m(f(\cdot+a))=m(f),
$$

(ii) for every $f \in \mathcal{B}(G, X)$ and closed convex bounded subset $V$ of $X$

$$
\operatorname{im}(f) \subset V \Longrightarrow m(f) \in V .
$$

There arises a natural question if for a given amenable group $(G,+)$ and a Banach space $X$, the space $\mathcal{B}(G, X)$ admits an invariant mean. Z. Gajda showed ([3, Theorem 2.3]) that the answer is true if the space $X$ is reflexive.

Theorem 1.4. Let $G$ be an amenable group, and let $X$ be a reflexive Banach space. Then $\mathcal{B}(G, X)$ admits an invariant mean.

Next, J. Tabor ([12, Theorem 1]) proves that invariant mean cannot be constructed for functions with values in non-reflexive Banach spaces (it was proved earlier by F. Bombal, G. Vera [2] in Spanish).

THEOREM 1.5. Let $X$ be an arbitrary non-reflexive Banach space. Then $\mathcal{B}(\mathbb{Z}, X)$ does not admit an invariant mean. 


\section{Notions}

In this work $(X,\|\cdot\|)$ is Banach space over the field $\mathbb{R}$. The map $\kappa: X \rightarrow$ $X^{* *}$ is given by

$$
\kappa(x)\left(x^{*}\right)=x^{*} x, x \in X, x^{*} \in X^{*} .
$$

We use some generalized definition of an invariant mean (see [4]).

Definition 2.1. Let $(S,+)$ be a left [right] amenable semigroup, $(X,\|\cdot\|)$ be a Banach space. A linear map $M: \mathcal{B}(S, X) \rightarrow X$ is called a left [right] invariant mean if

$$
\begin{gathered}
\|M\| \leq 1, \\
M\left(c 1_{S}\right)=c, \quad c \in X, \\
M\left({ }_{a} f\right)=M(f), \quad a \in S, f \in \mathcal{B}(S, X) \\
{\left[M\left(f_{a}\right)=M(f), \quad a \in S, f \in \mathcal{B}(S, X)\right],}
\end{gathered}
$$

where

$$
\begin{gathered}
{ }_{a} f(x)=f(a+x), \quad a, x \in S, f \in \mathcal{B}(S, X) \\
{\left[f_{a}(x)=f(x+a), \quad a, x \in S, f \in \mathcal{B}(S, X) .\right]}
\end{gathered}
$$

If $M$ is a left and right invariant mean, then $M$ is called an invariant mean.

It is easily seen that if $M: \mathcal{B}(S, X) \rightarrow X$ satisfies conditions of definition 1.3 , then $M$ satisfies conditions of definition 2.1 .

Definition 2.2. A Banach space $(X,\|\cdot\|)$ is said to have the invariant mean property if and only if for every left [right] amenable semigroup $(S,+)$ there exists a left [right] invariant mean $M: \mathcal{B}(S, X) \rightarrow X$.

We define some property which has been studied by Godefroy [5], Godefroy and Kalton [6], Rao [9].

Definition 2.3. A Banach space $X$ is said to be constrained in its bidual if $X$ is the range of a norm one linear projection when canonically embedded in its bidual $X^{* *}$. 
Definition 2.4. A Banach space $X$ has the finite-infinte intersection property if for every collection $\left\{B\left(x_{t}, r_{t}\right): t \in T\right\}$ of closed balls in $X$

$\left(I P_{f, \infty}\right) \quad\left(\forall J \subset T,|J|<\infty \bigcap_{t \in J} B\left(x_{t}, r_{t}\right) \neq \emptyset\right) \Longrightarrow \bigcap_{t \in T} B\left(x_{t}, r_{t}\right) \neq \emptyset$.

We use some part of a result from the work of Godefroy and Kalton 6, Corollary 6.6].

TheOrem 2.5. Let $X$ be a Banach space not containing $\ell_{1}$. The following conditions are equivalent:

(i) $X$ is a dual space.

(ii) $X$ satisfies $I P_{f, \infty}$.

(iii) $X$ is constrained in its bidual.

\section{Main result}

We start with some auxiliary theorem.

TheOREm 3.1. Let $(S,+)$ be a left [right] amenable semigroup and let $L: \mathcal{B}(S, \mathbb{R}) \rightarrow \mathbb{R}$ be a left [right] invariant mean. The map $\Lambda: \mathcal{B}(S, X) \rightarrow X^{* *}$ defined by the formula

$$
\Lambda(f)\left(x^{*}\right):=L\left(x^{*} \circ f\right), \quad x^{*} \in X^{*}, f \in \mathcal{B}(S, X),
$$

is linear, continuous and

$$
\begin{gathered}
\|\Lambda\| \leq 1, \\
\Lambda\left(c 1_{S}\right)=\kappa(c), \quad c \in X, \\
\Lambda\left(_{a} f\right)=\Lambda(f), \quad a \in S, f \in \mathcal{B}(S, X) \\
{\left[\Lambda\left(f_{a}\right)=\Lambda(f), \quad a \in S, f \in \mathcal{B}(S, X)\right] .}
\end{gathered}
$$

Proof. Assume that $(S,+)$ is a left amenable semigroup. Let $f, g \in$ $\mathcal{B}(S, X), \alpha, \beta \in \mathbb{R}, x^{*}, y^{*} \in X^{*}$. First we show that $\Lambda$ is well defined. Indeed, we have

$$
\begin{aligned}
\Lambda(f)\left(\alpha x^{*}+\beta y^{*}\right) & =L\left(\left(\alpha x^{*}+\beta y^{*}\right) \circ f\right)=L\left(\alpha x^{*} \circ f+\beta y^{*} \circ f\right) \\
& =\alpha L\left(x^{*} \circ f\right)+\beta L\left(y^{*} \circ f\right)=\alpha \Lambda(f)\left(x^{*}\right)+\beta \Lambda(f)\left(y^{*}\right),
\end{aligned}
$$


and

$$
\|\Lambda(f)\|=\sup \left\{\Lambda(f)\left(x^{*}\right):\left\|x^{*}\right\| \leq 1\right\}=\sup \left\{L\left(x^{*} \circ f\right):\left\|x^{*}\right\| \leq 1\right\} \leq\|f\| .
$$

The function $\Lambda$ is linear:

$$
\begin{aligned}
\Lambda(\alpha f+\beta g)\left(x^{*}\right) & =L\left(x^{*} \circ(\alpha f+\beta g)\right)=L\left(\alpha x^{*} \circ f+\beta x^{*} \circ g\right) \\
& =\alpha L\left(x^{*} \circ f\right)+\beta L\left(x^{*} \circ g\right)=\alpha \Lambda(f)\left(x^{*}\right)+\beta \Lambda(g)\left(x^{*}\right) .
\end{aligned}
$$

We have also

$$
\Lambda\left(c 1_{S}\right)\left(x^{*}\right)=L\left(x^{*} \circ\left(c 1_{S}\right)\right)=L\left(x^{*} c 1_{S}\right)=x^{*} c=\kappa(c)\left(x^{*}\right), \quad c \in X
$$

and

$$
\Lambda\left({ }_{a} f\right)\left(x^{*}\right)=L\left(x^{*} \circ_{a} f\right)=L\left({ }_{a}\left(x^{*} \circ f\right)\right)=L\left(x^{*} \circ f\right)=\Lambda(f)\left(x^{*}\right), \quad a \in S .
$$

The proof is analogous when $(S,+)$ is a right amenable semigroup.

Now we have a result which gives us a sufficient condition for the invariant mean property.

THEOREM 3.2. If $X$ is constrained in its bidual then the space $X$ has the invariant mean property.

Proof. Let $(S,+)$ be a left amenable semigroup, $\varphi: X^{* *} \rightarrow X$ be a norm one linear projection such that $\varphi \circ \kappa=i d_{X}$. Let further $\Lambda: \mathcal{B}(S, X) \rightarrow X^{* *}$ be a function from Theorem 3.1. We define the function $M: \mathcal{B}(S, X) \rightarrow X$ by the formula $M=\varphi \circ \Lambda$. It is easily seen that $M$ is linear and its norm is not great then 1 . We have also

$$
M\left(c 1_{S}\right)=\varphi\left(\Lambda\left(c 1_{S}\right)\right)=\varphi(\kappa(c))=c, \quad c \in X
$$

and

$$
M\left({ }_{a} f\right)=\varphi\left(\Lambda\left({ }_{a} f\right)\right)=\varphi(\Lambda(f))=M(f), \quad a \in S, f \in \mathcal{B}(S, X)
$$

The proof is analogous when $(S,+)$ is a right amenable semigroup.

Corollary 3.3. If $X$ is a reflexive space or has the Hahn-Banach extension property, then $X$ has the invariant mean property. 
Proof. If $X$ is a reflexive space then we take $\varphi=\kappa^{-1}$ in Theorem 3.2 . If $X$ has the Hahn-Banach extension property then we take $\varphi$ in Theorem 3.2 as an extension of $\kappa^{-1}: \kappa[X] \rightarrow X$ to the linear and continuous map on $X^{* *}$.

Corollary 3.4. If $(X,\|\cdot\|)$ is a normed space then the dual space $X^{*}$ has the invariant mean property.

Proof. Let $\kappa^{*}: X^{*} \rightarrow X^{* * *}, \varphi: X^{* * *} \rightarrow X^{*}$ be functions given by formulas

$$
\begin{gathered}
\kappa^{*}\left(x^{*}\right)\left(x^{* *}\right):=x^{* *} x^{*}, \quad x^{*} \in X^{*}, x^{* *} \in X^{* *}, \\
\varphi\left(x^{* * *}\right)(x):=x^{* * *} \kappa(x), \quad x \in X, x^{* * *} \in X^{* * *} .
\end{gathered}
$$

It is easy to see that $\varphi$ is well defined, it is linear and has norm not greater than 1 . We have also

$$
\varphi\left(\kappa^{*}\left(x^{*}\right)\right)(x)=\kappa^{*}\left(x^{*}\right)(\kappa(x))=\kappa(x)\left(x^{*}\right)=x^{*} x, \quad x \in X, x^{*} \in X^{*} .
$$

In view of Theorem 3.2 , the space $X^{*}$ has the invariant mean property.

Lemma 3.5. Let I be an infinite set, $S$ be a set defined by

$$
S=\left\{f: I \rightarrow\{0,1\}: \text { the set } f^{-1}(\{1\}) \text { is finite }\right\}
$$

with the operation $+: S \times S \rightarrow S$ given by the formula

$$
(f+g)(i)=\left\{\begin{array}{ll}
1, & f(i)=1 \vee g(i)=1, \\
0, & f(i)=g(i)=0,
\end{array} \quad i \in I, \quad f, g \in S .\right.
$$

Then $(S,+)$ is an abelian semigroup and $|S|=|I|$.

Proof. It is easy to see that the operation + is commutative. If we have $f, g, h \in S$, then

$$
\begin{aligned}
(f+(g+h))(i) & = \begin{cases}1, & f(i)=1 \vee g(i)=1 \vee h(i)=1 \\
0, & f(i)=g(i)=h(i)=0\end{cases} \\
& =((f+g))+h)(i), \quad i \in I .
\end{aligned}
$$


We have also

$$
|I| \leq|S| \leq\left|\bigcup_{n \in \mathbb{N}} I^{n}\right|=|\mathbb{N} \times I|=|I| .
$$

It is known that if $X$ is constrained in its bidual, then $X$ satisfies $I P_{f, \infty}$ and an inverse implication is an open problem. Theorem 3.2 and the corollary from the following theorem give us implications:

$X$ is constrained in its bidual $\Rightarrow X$ has the invariant mean property $\Rightarrow X$ satisfies $I P_{f, \infty}$.

Theorem 3.6. Let $X$ be a Banach space, $\left\{B\left(x_{i}, r_{i}\right): i \in I\right\}$ be a family of closed ball in $X$ such that

$$
\forall \text { } \subset I,|J|<\infty \bigcap_{i \in J} B\left(x_{i}, r_{i}\right) \neq \emptyset .
$$

Let further $(S,+)$ be a semigroup from Lemma 3.5. If there exists an invariant mean $M: \mathcal{B}(S, X) \rightarrow X$, then

$$
\bigcap_{i \in I} B\left(x_{i}, r_{i}\right) \neq \emptyset
$$

Proof. Fix $i_{0} \in I$. For every $J \subset I,|J|<\infty$ we take

$$
y_{J} \in \bigcap_{i \in J} B\left(x_{i}, r_{i}\right) \cap B\left(x_{i_{0}}, r_{i_{0}}\right) .
$$

We define $f: S \rightarrow X$ by the formula

$$
f(g)=y_{J} \Longleftrightarrow J=\{j \in I: g(j)=1\}, \quad g \in S .
$$

Since $y_{J} \in B\left(x_{i_{0}}, r_{i_{0}}\right)$ for every $J \subset I,|J|<\infty, f$ is bounded.

Let $M: \mathcal{B}(S, X) \rightarrow X$ be an invariant mean. Fix $k \in I$. Let $h=1_{\{k\}}$. Then $h \in S$ and

$$
(h+g)(k)=1, \quad g \in S .
$$

Hence

$$
k \in\{i \in I:(h+g)(x)=1\}, \quad g \in S,
$$


and

$$
{ }_{h} f(g)=f(h+g)=y_{\{i \in I:(h+g)(x)=1\}} \in B\left(x_{k}, r_{k}\right), \quad g \in S .
$$

We obtain

$$
M(f)=M\left({ }_{h} f\right) \in B\left(x_{k}, r_{k}\right),
$$

which means that

$$
M(f) \in \bigcap_{k \in I} B\left(x_{k}, r_{k}\right)
$$

showing that $\bigcap_{k \in I} B\left(x_{k}, r_{k}\right) \neq \emptyset$.

COROllary 3.7. If the Banach space $X$ has the invariant mean property then $X$ satisfies $I P_{f, \infty}$.

Now, in the view of Theorem 2.5, we can write the following result.

TheOREM 3.8. Let $X$ be a Banach space not containing $\ell_{1}$. The following conditions are equivalent:

(i) $X$ is a dual space.

(ii) $X$ is constrained in its bidual.

(iii) $X$ has the invariant mean property.

(iv) $X$ satisfies $I I P_{f, \infty}$.

Lemma 3.9. For every cardinal number $\gamma \geq 1$ there exists a commutative group $(G,+)$ such that $|G|=\gamma$.

Proof. If $\gamma$ is finite and $\gamma=n$ then we take $G=\mathbb{Z}_{n}$. If $\gamma$ is infinite and $A$ is a set of cardinality $\gamma$, then we define the set

$$
G:=\left\{\sum_{i=1}^{n} \varepsilon_{i} 1_{\left\{a_{i}\right\}}: \varepsilon_{1}, \ldots, \varepsilon_{n} \in \mathbb{Z}, a_{1}, \ldots, a_{n} \in A, n \in \mathbb{N}\right\} \subset \mathcal{B}(A, \mathbb{R}) .
$$

Observe that $(G,+)$ is a commutative group and

$$
|A| \leq|G| \leq\left|\bigcup_{n \in \mathbb{N}}(\mathbb{Z} \times A)^{n}\right|=\left|\bigcup_{n \in \mathbb{N}} A^{n}\right| \leq|A \times \mathbb{N}|=|A| .
$$


LEMma 3.10. Let $(G,+)$ be an uncountable group and $\gamma$ be a cardinal number such that $\aleph_{0} \leq \gamma \leq|G|$. There exists a subgroup $G_{0}$ of the group $G$ such that $\left|G_{0}\right|=\gamma$.

Proof. Let $\gamma$ be a cardinal number such that $\aleph_{0} \leq \gamma \leq|G|$. Let further $A \subset G$ be a set such that $|A|=\gamma$. We define

$$
G_{0}=\langle A\rangle=\left\{\sum_{i=1}^{n} a_{i}: a_{i} \in \tilde{A}, n \in \mathbb{N}\right\}=\bigcup_{n \in \mathbb{N}} \underbrace{\tilde{A}+\ldots+\tilde{A}}_{n},
$$

where $\tilde{A}=A \cup-A$. Hence

$$
\left|G_{0}\right|=|\bigcup_{n \in \mathbb{N}} \underbrace{\tilde{A}+\ldots+\tilde{A}}_{n}|=\left|\bigcup_{n \in \mathbb{N}} \tilde{A}^{n}\right|=|\mathbb{N} \times \tilde{A}|=|A|=\gamma .
$$

REMARK 3.11. We have the same result for the semigroup: If $(S,+)$ is an uncountable semigroup and $\gamma$ is a cardinal number such that $\aleph_{0} \leq \gamma \leq|S|$, then there exists a subsemigroup $S_{0}$ of the semigroup $S$ such that $\left|S_{0}\right|=\gamma$.

The next theorem shows that if an invariant mean exists for some abelian group, then an invariant mean exists on each subgroup of this group.

Theorem 3.12. Let $(G,+)$ be a group, $(X,\|\cdot\|)$ be a Banach space. Assume that there exists a left [right] invariant mean $M: \mathcal{B}(G, X) \rightarrow X$. Then, for every subgroup $G_{0}$ of the group $G$, there exists a left [right] invariant mean $M: \mathcal{B}\left(G_{0}, X\right) \rightarrow X$.

Proof. Assume that there exists a left invariant mean $M: \mathcal{B}(G, X) \rightarrow X$ (for a right invariant mean the proof is similar). Let $G_{0}$ be a subgroup of the group $G$. We have the right coset of $G_{0}$ in $G$ :

$$
G \backslash G_{0}=\left\{G_{0}+g_{\alpha}: g_{\alpha} \in G, \alpha \in A\right\},
$$

where $\alpha \neq \beta \Longrightarrow g_{\alpha}+G_{0} \neq g_{\beta}+G_{0}$. We define $M_{0}: \mathcal{B}\left(G_{0}, X\right) \rightarrow X$ by the formula

$$
M_{0}\left(f_{0}\right)=M\left(\psi\left(f_{0}\right)\right), \quad f_{0} \in \mathcal{B}\left(G_{0}, X\right),
$$

where $\psi: \mathcal{B}\left(G_{0}, X\right) \rightarrow \mathcal{B}(G, X)$ is given by

$$
\psi\left(f_{0}\right)\left(g_{0}+g_{\alpha}\right)=f_{0}\left(g_{0}\right), \quad f_{0} \in \mathcal{B}\left(G_{0}, X\right), g_{0} \in G_{0}, \alpha \in A .
$$


We notice that $\left.\left.\psi\left({ }_{a} f_{0}\right)\right)={ }_{a} \psi\left(f_{0}\right)\right), a \in G_{0}, f_{0} \in \mathcal{B}\left(G_{0}, X\right)$. Indeed we have

$$
\begin{aligned}
\psi\left({ }_{a} f_{0}\right)\left(g_{0}+g_{\alpha}\right) & ={ }_{a} f_{0}\left(g_{0}\right)=f_{0}\left(a+g_{0}\right)=\psi\left(f_{0}\right)\left(a+g_{0}+g_{\alpha}\right) \\
& ={ }_{a} \psi\left(f_{0}\right)\left(g_{0}+g_{\alpha}\right), \quad f_{0} \in \mathcal{B}\left(G_{0}, X\right), \quad a, g_{0} \in G_{0}, \alpha \in A .
\end{aligned}
$$

We will show that $M_{0}$ is an invariant mean. The linearity of $M_{0}$ follows from the linearity of $M$ and $\psi$. Since $\|\psi\|=1$, we obtain that $\left\|M_{0}\right\| \leq\|M\|=1$. We have also

$$
\begin{aligned}
M_{0}\left(c 1_{G_{0}}\right) & =M\left(c 1_{G}\right)=c, \quad c \in X, \\
M_{0}\left({ }_{a} f_{0}\right) & =M\left(\psi\left({ }_{a} f_{0}\right)\right)=M\left({ }_{a} \psi\left(f_{0}\right)\right)=M\left(\psi\left(f_{0}\right)\right) \\
& =M_{0}\left(f_{0}\right), \quad f_{0} \in \mathcal{B}\left(G_{0}, X\right), a \in G_{0} .
\end{aligned}
$$

Now we prove that if we have an invariant mean on some semigroup, then sets of cardinality less than cardinality of this semigroup do not change the value of this mean.

Lemma 3.13. Let $(S,+)$ be an infinite right cancellative semigroup, $(X,\|\cdot\|)$ be a normed space, and $\mu: 2^{S} \rightarrow X$ be a mapping such that

$$
\begin{gathered}
\|\mu(D)\| \leq 1, \quad D \subset S, \\
\mu(A \cup B)=\mu(A)+\mu(B), \quad A, B \subset S, A \cap B=\emptyset, \\
\mu\left({ }_{t} D\right)=\mu(D), \quad t \in S, D \subset S,
\end{gathered}
$$

where

$$
{ }_{t} D:=\{s \in S: t+s \in D\}, t \in S, D \subset S .
$$

Then, for every set $D \subset S$ such that $|D|<|S|$, we have $\mu(D)=0$.

Proof. Let $D \subset S$ be such that $|D|<|S|$. Suppose that

$$
\forall_{t \in S} D \cap{ }_{t} D \neq \emptyset \text {. }
$$

Then for every $t \in S$ there exist some $s_{t} \in D$ such that $t+s_{t} \in D$. We define sets $A_{s}:=\left\{t \in S: s_{t}=s\right\}$. Observe that $S=\bigcup_{s \in D} A_{s}$. Thus there exists $s_{0} \in D$ such that $|D|<\left|A_{s_{0}}\right|$. Let $\varphi: A_{s_{0}} \rightarrow D$ be the map given by

$$
\varphi(t)=t+s_{0}, \quad t \in A_{s_{0}} .
$$


Since $(S,+)$ is a right cancellative semigroup, we obtain that $\varphi$ is one-to-one and we have $\left|A_{s_{0}}\right| \leq|D|$, which is a contradiction. Thus there exist $t \in S$ such that $D \cap{ }_{t} D=\emptyset$. Since $\left|D \cup{ }_{t} D\right|=|D|$, it follows from above consideration that there exists a sequence $\left(t_{n}\right)_{n \in \mathbb{N}}$ of elements $S$ such that

$$
\left(D \cup \bigcup_{k=1}^{n} t_{k} D\right) \cap t_{t_{n+1}} D=\emptyset, \quad n \in \mathbb{N} .
$$

Hence we have

$$
1 \geq \mu\left(\bigcup_{k=1}^{n} t_{k} D\right)=\sum_{k=1}^{n} \mu\left(t_{k} D\right)=n \mu(D), \quad n \in \mathbb{N},
$$

which implies that $\mu(D)=0$.

Since each normed space is isometrically isomorphic to some subspaces of the space $\mathcal{B}(\Omega, \mathbb{R})$ with the supremum norm (we can take as $\Omega$ a subset of all norm one vectors of $X^{*}$ and it is easy to check that $\varphi$ given by the formula $\varphi(x)\left(x^{*}\right)=x^{*} x, x \in X, x^{*} \in \Omega$, is an isometric isomorphism of $X$ onto $\varphi(X))$, we may restrict our investigation to such subspaces.

TheOREm 3.14. Let $\Omega$ be an infinite set, $(X,\|\cdot\|)$ be a subspace of space $\mathcal{B}(\Omega, \mathbb{R})$ such that

$$
\begin{gathered}
1_{\{\omega\}} \in X, \quad \omega \in \Omega, \\
\|f\|=\sup _{\omega \in \Omega}|f(\omega)|, \quad f \in X .
\end{gathered}
$$

Let further $(G,+)$ be a group. If there exists a left (or right) invariant mean $M: \mathcal{B}(G, X) \rightarrow X$, then $1_{A} \in X$ for all $A \subset \Omega$ such that $|A| \leq|G|$.

In particular, if the space $(X,\|\cdot\|)$ has the invariant mean property then $X=\mathcal{B}(\Omega, \mathbb{R})$.

Proof. Let $(G,+)$ be a group. Suppose that there exists $Z \subset \Omega,|Z| \leq$ $|G|$, such that $1_{Z} \notin X$. Since every bounded function from $\Omega$ to $\mathbb{R}$ is the uniformly limit of simple functions, there exists $A \subset Z$ such that $1_{A} \notin X$ and

$$
\forall_{B \subset \Omega}\left(|B|<|A| \Rightarrow 1_{B} \in X\right) .
$$

Since $1_{\{\omega\}} \in X, \omega \in \Omega$, we obtain that $1_{B} \in X$ for all $B \subset \Omega,|B|<\aleph_{0}$, so we may assume that $(G,+)$ is infinite. Let $M: \mathcal{B}(G, X) \rightarrow X$ be a left (or right) invariant mean. In view of Lemma 3.10 and Theorem 3.12 , we may assume 
that $|G|=|A|$. Let further $(P,<)$ be a set of all ordinal numbers less then $|A|, \varphi: A \rightarrow P, \psi: G \rightarrow P$ be bijections. We define sets

$$
B_{s}:=\{\omega \in A: \varphi(\omega)<\psi(s)\}, \quad s \in G .
$$

Observe that

$$
\left|B_{s}\right| \leq \psi(s)<|G|=|A| .
$$

We define the function $f: G \rightarrow \mathcal{B}(\Omega, \mathbb{R})$ by the formula

$$
f(s):=1_{B_{s}}, \quad s \in G .
$$

Notice that $f \in \mathcal{B}(G, X)$. We define sets

$$
C_{\omega}:=\left\{s \in G: \omega \in B_{s}\right\}, \omega \in A .
$$

Hence we have

$$
\begin{aligned}
\left|G \backslash C_{\omega}\right| & =\left|\left\{s \in G: \omega \notin B_{s}\right\}\right|=|\{s \in G: \psi(s) \leq \varphi(\omega)\}| \\
& \leq \varphi(\omega)+1<|A|=|G|, \quad \omega \in A .
\end{aligned}
$$

In view of Lemma 3.13 , we obtain

$$
M\left(1_{\{\omega\}} 1_{G \backslash C_{\omega}}\right)=0, \quad \omega \in A,
$$

and thus

$$
M\left(1_{\{\omega\}} 1_{C_{\omega}}\right)=M\left(1_{\{\omega\}} 1_{G}\right)=1_{\{\omega\}}, \quad \omega \in A .
$$

For $\omega \in \Omega \backslash A$ we have

$$
\begin{aligned}
\left\|M(f)-1_{\{\omega\}}\right\| & =\left\|M\left(f-1_{\{\omega\}} 1_{G}\right)\right\| \leq\left\|f-1_{\{\omega\}} 1_{G}\right\|=1, \\
\left\|M(f)+1_{\{\omega\}}\right\| & =\left\|M\left(f+1_{\{\omega\}} 1_{G}\right)\right\| \leq\left\|f+1_{\{\omega\}} 1_{G}\right\|=1,
\end{aligned}
$$

hence

$$
M(f)(\omega)=0, \quad \omega \in \Omega \backslash A .
$$


For $\omega \in A$ we have

$$
\begin{aligned}
\|M(f)\| & \leq\|f\|=1 \\
\left\|M(f)-2 \cdot 1_{\{\omega\}}\right\| & =\left\|M(f)-2 M\left(1_{\{\omega\}} 1_{C_{\omega}}\right)\right\|=\left\|M\left(f-2 \cdot 1_{\{\omega\}} 1_{C_{\omega}}\right)\right\| \\
& \leq\left\|f-2 \cdot 1_{\{\omega\}} 1_{C_{\omega}}(s)\right\|=\sup _{s \in G}\left\|1_{B_{s}}-2 \cdot 1_{\{\omega\}} 1_{C_{\omega}}\right\| \\
& =\sup _{s \in G a \in A}\left|1_{B_{s}}(a)-2 \cdot 1_{\{\omega\}}(a) 1_{C_{\omega}}(s)\right|=1
\end{aligned}
$$

hence

$$
M(f)(\omega)=1, \omega \in A
$$

Finally we have $M(f)=1_{A} \notin X$, which is an obvious contradiction.

Corollary 3.15. The spaces $c$ and $c_{0}$ do not have the invariant mean property.

ExAmple 3.16 . The space $C_{B}(\mathbb{R})$ does not have the invariant mean property.

To prove the claim let $X=C_{B}(\mathbb{R})$ and suppose that there exists an invariant mean $M: \mathcal{B}(\mathbb{N}, X) \rightarrow X$. First we show that if $f \in \mathcal{B}(\mathbb{N}, X)$ and $f(n)(x) \geq 0, x \in \mathbb{R}$, then $M(f)(x) \geq 0, x \in \mathbb{R}$. Indeed, we have

$$
\begin{aligned}
\|M(f)-\| f\left\|\cdot 1_{\mathbb{R}}\right\| & =\left\|M\left(f-\|f\| \cdot 1_{\mathbb{R}} 1_{\mathbb{N}}\right)\right\| \\
& \leq\|f-\| f\left\|\cdot 1_{\mathbb{R}} 1_{\mathbb{N}}\right\| \leq\|f\|,
\end{aligned}
$$

and consequently

$$
M(f)(x)-\|f\| \geq-\|f\|, \quad x \in \mathbb{R}
$$

For $n \in \mathbb{N}$ we define functions:

$$
f_{n}(x):=\left\{\begin{array}{ll}
0, & x \leq 0, \\
n x, & x \in\left(0, \frac{1}{n}\right), \\
1, & x \geq \frac{1}{n},
\end{array} \quad g_{n}(x):= \begin{cases}0, & x \leq-\frac{1}{n} \\
1+n x, & x \in\left(-\frac{1}{n}, 0\right) \\
1, & x \geq 0\end{cases}\right.
$$

Let $f(n):=f_{n}, n \in \mathbb{N}$. Then $f \in \mathcal{B}(\mathbb{N}, X)$. Observe that

$$
f_{i}(x) \leq f(n)(x) \leq g_{k}(x), \quad i, k, n \in \mathbb{N}, i \leq n, x \in \mathbb{R}
$$


Hence

$$
f_{n} \leq M(n f)=M(f) \leq g_{n}
$$

and thus

$$
\begin{aligned}
& M(f)(x)=0, x<0, \\
& M(f)(x)=1, x>0,
\end{aligned}
$$

which contradicts the continuity of $M(f)$.

\section{References}

[1] Badora R., Ger R., Páles Zs., Additive selections and the stability of the Cauchy functional equation, ANZIAM J. 44 (2003), 323-337.

[2] Bombal F., Vera G., Means in locally convex spaces and semireflexivity, Collect. Math. 24 (1973), 267-295 (in Spanish).

[3] Gajda Z., Invariant means and representations of semigroups in the theory of functional equations, Wydawnictwo Uniwersytetu Slaskiego, Katowice, 1992.

[4] Ger R., The singular case in the stability behavior of linear mappings, Grazer Math. Ber. 316 (1992), 59-70.

[5] Godefroy G., Five lectures in Geometry of Banach spaces, Seminar on Functional Analysis (1987), 9-67.

[6] Godefroy G., Kalton N.J., The ball topology and its application, Contemp. Math. 85 (1989), 195-237.

[7] Greenleaf F.P., Invariant means on topological groups and their applications, Van Nostrand Mathematical Studies, No. 16, Van Nostrand Reinhold Co., New York-TorontoLondon-Melbourne, 1969.

[8] Hewitt E., Ross K., Abstract harmonic analysis. Vol. I, Academic Press, New York, 1962.

[9] Rao T.S.S.R.K., $L^{1}(\mu, X)$ as a constrained subspace of its bidual, Proc. Indian Acad. Sci. Math. Sci. 109 (1999), no. 3, 309-315.

[10] Székelyhidi L., A note on Hyers theorem, C. R. Math. Rep. Acad. Sci. Canada 8 (1986), $127-129$.

[11] Tabor J., Monomial selections of set-valued functions, Publicationes Math. Debrecen 56 (2000), no. 1-2, 33-42.

[12] Tabor J., Note on reflexivity and invariant means, Univ. Iagel. Acta Math. 43 (2005), 99-102.

Institute of Mathematics

UNIVERSITY OF Silesia

BANKOWA 14

40-007 Katowice

PolAnd

e-mail: rlukasik@math.us.edu.pl 